\title{
Politique
}

\section{La politique technologique québécoise}

\section{Louise-E. Fortin}

Numéro 8, automne 1985

Innovations et politiques technologiques

URI : https://id.erudit.org/iderudit/040496ar

DOI : https://doi.org/10.7202/040496ar

Aller au sommaire du numéro

Éditeur(s)

Société québécoise de science politique

ISSN

0711-608X (imprimé)

1918-6584 (numérique)

Découvrir la revue

Citer cet article

Fortin, L.-E. (1985). La politique technologique québécoise. Politique, (8), 23-44. https://doi.org/10.7202/040496ar d'utilisation que vous pouvez consulter en ligne.

https://apropos.erudit.org/fr/usagers/politique-dutilisation/ 


\title{
LA POLITIQUE TECHNOLOGIQUE QUÉBECOISE*
}

\author{
Louise-E. Fortin \\ Université de Montréal
}

L'idée que le progrès technique stimule la croissance économique n'est plus contestée. Même l'idée qu'il serait le plus lourd des facteurs de croissance est largement répandue ( $\mathrm{R}$. Rothwell et W. Zegveld, 1981, p. 26). Par ailleurs il est raisonnable de penser que la recherche et développement (R-D), sans qu'elle soit pour autant unique, demeure l'activité la plus déterminante du progrès technique (Unesco, 1971 p. 34 et C. Freeman, 1971, p. 237).

Quand à savoir si l'intervention étatique dans le développement technique et l'intensification de l'effort de recherche est pertinente, la question est superflue dans la mesure où il est dans l'intérêt de l'État de favoriser la croissance économique. Par contre la question du «comment» intervenir est plus difficile à résoudre. L'État en effet, doit-il soutenir, stimuler ou même provoquer les changements technologiques? Voilà bien un terrain d'expérimentations sur lequel aucun gouvernement n'est engagé sans une certaine inquiétude.

\footnotetext{
* Cet article est issu d'une recherche intitulée «Importations de technologie et politique gouvernementales» dirigée par $\mathrm{G}$. Boismenu et $\mathrm{G}$. Ducatenzeiler du département de science politique de l'Université de Montréal, et financée par le Conseil de recherche en sciences humaines du Canada.
} 
Notre propos est de faire le point sur l'expérience québécoise plus récente et plus modeste que celle du gouvernement fédéral, mais pour l'heure, intensive et déterminée. Nous ferons l'examen des objectifs et des moyens mis en œuvre afin de corriger un état de choses jugé insatisfaisant. Nous savons que le Québec a un faible potentiel technologique et consacre relativement peu d'effort à la recherche et développement; qu'il suffise de mentionner qu'en 1981, 1\% seulement de son PIB était investi dans la R-D; en 1979, le Québec se classait au douzième rang, après le Canada et avant l'Italie, parmi les principaux pays de l'OCDE au titre de l'investissement en R-D (L. Séguin-Dulude et R. Lacroix, 1983 , p. 43). Et s'il est besoin de frapper davantage les esprits, ajoutons que le Québec pays peu peuplé, y consacrait 93 \$ per capita en 1979, alors qu'en moyenne les douze principaux pays de l'OCDE investisseurs en R-D dépensaient 183 \$ (ibid p. 43).

L'importance de la mainmise du capital étranger sur l'industrie, c'est-à-dire d'entreprises qui généralement importent leur technologie de leur société-mère (J. Britton et J. Gilmour, 1980), la petite taille des entreprises autochtones qui n'ont pas les moyens financiers de faire de la recherche, le profond déséquilibre de la structure industrielle dominée par l'industrie primaire et l'industrie manufacturière à faible intensité technologique, auxquels s'ajoute l'obstacle d'un marché local étroit, tout cela explique le faible potentiel technologique de l'industrie québécoise et sa faible propension à faire de la recherche. Par exemple en 1979, celle-ci ne dépensait que $0,50 \%$ du PIB québécois dans la recherche, loin derrière la performance suèdoise $(1,80 \%)$, suisse $(1,31 \%)$, japonaise $(1,22 \%)$, proche de celle de l'Italie $(0,49 \%)$ de l'Australie $(0,24 \%)$ et du Canada dans son ensemble $(0,48 \%)$ (ibid p. 66).

Bref des politiques technologiques sont requises, c'est-àdire des politiques dont la spécificité dans une stratégie industrielle est d'encourager le progrès des connaissances de façon à soutenir le progrès technique (G. Boismenu et G. Ducatenzeiler, 1984, 
Introduction). Et ce sont elles qui retiennent notre attention: les politiques technologiques énoncées et mises en œuvre à partir du début des années 70 jusqu'à 1983 environ. Nous tiendrons compte du discours dans la mesure où il devrait nous permettre de repérer les priorités sectorielles, d'analyser la distance entre ce qui est dit et ce qui est fait et, à un niveau plus général, de saisir la philosophie qui sous-tend les actions. Puis dans la perspective de l'analyse que nous faisons du faible potentiel technologique québécois, nous examinerons deux formes d'interventions: 1- les politiques de promotion directe de la $\mathrm{R}-\mathrm{D}$ par le moyen d'une aide financière directe à l'industrie ou l'implantation d'une infra-structure de recherche industrielle. 2- les barrières non-tarifaires telles les politiques de contrôle des investissements étrangers sur le plan de leur impact technologique et les politiques de protection des marchés publics au profit des produits «à contenu» québécois. Tout cela dans le contexte d'une stratégie industrielle et commerciale qui devrait fixer les objectifs sectoriels, travailler à la correction de la structure industrielle dans le sens du développement d'une industrie à plus forte intensité technologique et rendre les produits québécois accessibles aux marchés internationaux de façon à compenser les effets de l'étroitesse du marché local sur la capacité d'innovation de l'industrie.

\section{Le discours gouvernemental}

La préoccupation du gouvernement québécois en matière de politique technologique remonte à la fin des années soixante, mais jusqu'à la fin des années soixante-dix, elle n’a pas donné lieu à des actions très soutenues et coordonnées. Jusqu'en 1976, l'Université plutôt que l'industrie ou le gouvernement, détient une forte emprise sur le système de recherche québécois y compris la recherche industrielle. L'Institut national de recherche scientifique (INRS), le Centre de recherche industrielle (CRIQ), le Centre de 
recherche minérale (CRM), le Complexe scientifique de Ste-Foy, tous ces organismes mis sur pied autour de 1970 afin de favoriser la recherche appliquée, la recherche technologique et directement la recherche industrielle, sont tous à des degrés différents, formellement ou informellement, rattachés à l'Université du Québec nouvellement créée. Le choix n'est pas indifférent. Les conséquences les plus évidentes se traduisent par une production programmée de l'intérieur sans lien avec les besoins de l'industrie ni bien sûr avec une politique technologique et industrielle énonçant des objectifs sectoriels. D'où l'échec relatif et la remise en question. L'année 1976 marque un virage. Dans son livre vert sur la Politique québécoise du développement culturel, le gouvernement indique ses intentions d'harmoniser le choix des domaines scientifiques et technologiques aux objectifs de la société (ministère d'État au Développement Culturel, 1978, p. 277). Cette simple manière de penser est significative. Elle vient marquer la fin de l'ère du «laisser-faire». Le livre blanc qui suivra établira quelques modalités des transformations à mettre en ouvre, notamment un nouveau mode de relation entre les divers intervenants ( $U n$ projet collectif, 1980).

Or le développement économique, sans contredit la priorité gouvernementale des années 80 , passe par le développement du potentiel technologique, de la capacité d'innovation de l'industrie et l'intensification de l'effort de recherche. C'est ainsi que le document Bâtir le Québec reconnait la nécessité de mettre l'accent sur la recherche et l'innovation par les moyens suivants: «...la promotion de l'innovation, l'amélioration de la qualité et de l'efficacité des ressources humaines hautement qualifiées par l'établissement de liens privilégiés avec l'Université; l'augmentation des ressources humaines et des ressources en capital par le moyen de programmes d'aide directe; l'amélioration des ressources externes à l'industrie, l'information technologique et enfin, l'offre de services de recherche à assurer par le renforcement du Centre de recherche 
industrielle et d'autres institution publiques spécialisées (IREQ, CRM, etc.) (ministère d'État au Développement Économique, 1980, p. 175).

Enfin le document le Virage technologique (Ministère d'État au Développement Économique, 1982) programme d'action économique 1982-1986, appuie le développement économique sur le développement technique et technologique et propose des priorités sectorielles: le tourisme, l'agro-alimentaire, la forêt, l'énergie, les mines et le transport, l'informatique-électronique et les biotechnologies.

De tous ces discours sur le développement technologique produit par le gouvernement québécois depuis la fin des années soixante-dix, il importe surtout de retenir l'apparition d'un esprit radicalement nouveau; celui qui animait déjà l'OCDE au cours des années soixante, à savoir que ce sont désormais les impératifs du marché qui devront dicter les objectifs de la recherche technologique; d'où la place centrale de l'industrie, d'où la nécessité de transformer la rationalité institutionnelle de l'infra-structure scientifico-technologique notamment celle de l'Université laquelle devra maintenant chercher à intégrer davantage le développement des ressources humaines et de la recherche aux besoins de l'industrie. Quant à y trouver une politique sectorielle bien articulée à des moyens d'action conséquents, le discours demeure vague et il vaut mieux examiner les actions effectives. D'abord le gouvernement québécois a mis l'accent sur la promotion directe de la recherche et développement par le moyen de transferts directs vers l'industrie sous forme d'avantages fiscaux, de prêts, garanties de prêts, acquisitions d'actions ou subventions, et par l'implantation d'une infra-structure de recherche publique et para-publique.

\section{La politique fiscale et la R-D dans l'industrie}

L'établissement d'une politique fiscale dans le but d'encourager le développement de la recherche dans l'industrie date du début 
des années soixante-dix. En vertu de la Loi favorisant le développement industriel au moyen d'avantages fiscaux (L.Q. 1971, chap. 23), loi administrée par la Société de Développement Industriel (SDI), est exempté d'impôts et de la taxe de vente, tout nouvel investissement dans le secteur manufacturier. En vertu de la loi de l'impôt (L.Q. 1972, chap. 23) toute entreprise qui s'adonne à des travaux de R-D peut déduire de ses revenus toutes dépenses afférentes encourues dans l'année, y compris les dépenses d'immobilisations. La loi exempte aussi de l'impôt les subventions accordées en vertu de la loi stimulant la R-D (IRDIA), au sens où ces subventions ne réduisent pas le montant de l'investissement aux fins du calcul de l'impôt; enfin elle exempte les corporations constituées exclusivement pour faire de la recherche.

Le fonds de relance industrielle créé en 1977, dont le financement provient d'entreprises qui acceptent d'y verser $50 \%$ de l'impôt qui serait autrement versé au Québec, rembourse à l'entreprise qui en bénéficie, $25 \%$ des dépenses admissibles, notamment les dépenses de R-D ou les dépenses occasionnées par l'acquisition de brevets ou de licences. Et depuis 1982, suite à la modification de la loi de la SDI dans le sens du nouveau programme d'action économique du gouvernement québécois $1982-$ 1986, il est possible d'obtenir un crédit d'impôt équivalent à $10 \%$ de la masse salariale affectée à la R-D. La loi modifiée fait aussi en sorte que toute personne qui procède à des dons destinés à une fondation pour le développement de la science et de la technologie, permet à ses héritiers d'obtenir un crédit d'impôt de $90 \%$ contre les droits successoraux à payer. (ministère de la Science et de la Technologie, 1983a, p. 17).

En 1983-1984, l'ensemble des dispositions fiscales reliées à la R-D, a coûté $26 \mathrm{M} \$$ au gouvernement, soit l'une des mesures les plus onéreuses en vue de soutenir le développement de la $\mathrm{R}$ D. (ibid, p. 23 à 26). Conjuguée avec l'action du gouvernement fédéral qui met de plus en plus l'accent sur cette forme d'aide à 
l'industrie (Marc Lalonde, 1983, tableau 1.7, p. 7), il est possible que le Québec soit parmi les tous premiers lieux au monde où l'impact des mesures fiscales reliées à la $\mathrm{R}-\mathrm{D}$ est le plus bénéfique (L. Séguin-Dulude et R. Lacroix, 1983, p. 120). Cependant il est difficile de mesurer cet impact et notamment de savoir qui en tire bénéfice. Une analyse produite par le gouvernement fédéral au niveau de l'industrie canadienne dans son ensemble, peut fournir un indice de l'effet des allègements fiscaux sur l'industrie québécoise. Cette analyse montre qu'en 1980 par exemple, une forte proportion des investissements dans la R-D, 31\%, était effectuée par des entreprises qui ne pouvaient se prévaloir des avantages fiscaux simplement parce qu'elles n'étaient pas imposables; $38 \%$ de ces investissements provenaient d'entreprises qui ne pouvaient utiliser la totalité de leur crédit d'impôt à la R-D dans l'année. Dans l'ensemble $69 \%$ des fonds affectés à la R-D étaient utilisés par des entreprises qui ne pouvaient tirer un profit maximum des dispositions du régime fiscal (M. Lalonde, op. cit., p. 8). Cette analyse est un indice des effets de la politique fiscale québécoise, avantageuse pour qui en profite dans la mesure où elle est peu contraignante, mais qui certainement exclut de ses bénéfices une forte proportion d'entreprises déjà engagées ou qui voudraient s'engager dans la recherche. Elle suppose d'autres moyens d'action.

Les prêts, garanties de prêts, acquisitions d'actions ou subventions, et la $\mathrm{R}-\mathrm{D}$ dans l'industrie.

Contrairement aux politiques fiscales, les politiques de financement de la R-D sous forme de prêts, garanties de prêts, acquisitions d'actions ou subventions, sont récentes au Québec. Elles ont été pendant longtemps l'affaire du gouvernement fédéral. Mais il reste qu'indirectement, certaines actions menées depuis une quinzaine d'années, ont stimulées le développement technologique ou la propension à l'innovation, au sens où elles ont 
contribuées à la modernisation d'entreprises québécoises et au développement d'une industrie à plus forte intensité technologique. Nous pensons aux actions de la Société de Développement Industriel et à celles de la Société Générale de Financement.

La SDI créé en 1971, constitue un des principaux outils financiers dont s'est doté le gouvernement dans le but explicite de moderniser la structure industrielle québécoise. Au départ la Société administrait trois programmes dont le programme d'aide aux entreprises à technologie moderne, le plus susceptible d'encourager le développement technologique.

Aujourd'hui la SDI administre neuf programmes dont trois devraient avoir un impact sur le potentiel technologique québécois. Il s'agit du programme d'aide à l'investissement manufacturier favorisant l'implantation d'entreprises "à technologie moderne» et d'entreprises «dynamiques». Sa vocation est d'encourager le développement d'une industrie innovatrice. Le financement de ce programme constitue la mesure la plus coûteuse, $41 \mathrm{M} \$$ ou $19 \%$ des $218 \mathrm{M} \$$ affectés à cette fin par le gouvernement québécois (ministère de la Science et de la Technologie, 1983a, p. 23 à 26).

Créé en 1982 dans la suite des dispositions du programme d'action économique 1982-1986, le programme d'aide aux activités de recherche et d'innovation (PARIQ) a pour mission de financer la recherche dans l'industrie par le moyen de prêts sans intérêt remboursables par redevance s'il y a succès. Le montant prêté peut atteindre $90 \%$ des dépenses admissibles, déduction faite de l'aide fédérale. Entre janvier 1983 et février 1984, le PARIQ a soutenu 42 projets dont deux recevaient plus d'un millions $\$$, trois plus de $500000 \$$ et cinq entre $200000 \$$ et $500000 \$$ (chiffres fournis par la SDI).

Le programme d'aide à l'investissement pour les laboratoires de recherche soutient les entreprises à but lucratif des secteurs secondaire et tertiaire qui exploitent un laboratoire de recherche appliquée. Pour bénéficier d'une aide, le programme exige que l'activité 
principale du laboratoire crée des biens ou services commerciaux.

Dans quelle mesure l'action de la SDI respecte-elle son objectif initial (celui de contribuer à la modernisation de la structure industrielle québécoise et favoriser le développement d'une industrie à plus forte intensité technologique) ou aux priorités sectorielles retenues par le nouveau programme d'action économique du gouvernement (l'agro-alimentaire, la forêt, les mines l'énergie, le transport et le tourisme)?

Après dix ans d'opération, le 31 mars 1980, la SDI avait distribué sous forme de prêts, garanties de prêts, acquisitions d'actions ou subventions, par le biais de son programme d'aide aux entreprises à technologie moderne surtout, 3069 aides pour la somme de $837 \mathbf{M}$ \$, dont $199 \mathbf{M} \$(23 \%)$ sont allés à l'industrie du matériel de transport, (le matériel de transport terrestre surtout), $69 \mathrm{M} \$$ $(8 \%)$ à celle des aliments et boissons, $64 \mathrm{M} \$(7,5 \%)$ aux produits du bois et $50 \mathrm{M} \$(5 \%)$ à l'industrie des appareils et du matériel électrique. (SDI, 1981).

Pour estimer la pertinence de ces interventions par rapport à l'objectif initial de la SDI ou à leur impact technologique, notons qu'en 1978 par exemple, l'industrie du matériel de transport, classée parmi les industries à forte et moyenne intensité technologique (Statistiques Canada, cat. 88-201, 1982-1983), effectuait $31 \%$ des dépenses de R-D industrielle intra-muros (Secrétariat du Développement Scientifique, 1982 tableau 4 p. 21) et contribuait à $6 \%$ de la valeur ajoutée manufacturière québécoise (Annuaire du Québec 1979-80, tableau 4, p. 999). Elle a reçu $23 \%$ de l'aide distribuée par la SDI. L'industrie des aliments et boissons, classée parmi les industrie à faible intensité technologique, a effectué $3 \%$ des dépenses de R-D seulement mais contribué à $13 \%$ de la valeur ajoutée manufacturière. Elle a reçu $8 \%$ de l'aide: L'industrie des produits du bois, à moyenne ou à faible intensité technologique, a fourni $8 \%$ des dépenses de R-D et $16 \%$ de la valeur ajoutée manufacturière. Elle a reçu $7,5 \%$ de l'aide. L'industrie 
des produits métalliques, à moyenne intensité technologique, a contribué à $2 \%$ seulement des dépenses de R-D et $7 \%$ de la valeur ajoutée manufacturière. Elle a reçu $7 \%$ de l'aide

Par rapport à sa contribution aux dépenses de R-D, 12\%, l'industrie des produits chimiques (drogues et médicaments et autres), à moyenne intensité technologique, semble avoir été négligée. Par contre l'industrie des aliments et boissons et celle des produits métalliques auraient été relativement trop privilégiées si on tient compte de leur performance faible technologique.

Comparée à l'action du gouvernement fédéral menée au Québec entre 1975 et 1982 dans le cadre de ses trois programmes d'aide à l'innovation (le programme d'expansion des entreprises, le programme d'aide à la recherche industrielle et le programme de productivité du matériel de défense), celle-ci favorisait surtout et de loin l'industrie du matériel de transport (avions et pièces), puis celle des appareils et du matériel électriques et celle des machines (ministère de la Science et de la Technologie, 1983b).

Quant à savoir si aujourd'hui la SDI respecte les priorités sectorielles proposées par le programme d'action économique actuel, les données sont encore peu significatives. En tout cas en 19821983, le SDI a distribué 781 aides pour une somme globale de $181 \mathrm{M} \$$, dont $66 \%$ sont allés à l'industrie des produits métalliques, $9 \%$ à l'industrie des appareils et matériel électriques, $9 \%$ à celle des produits du bois et $6 \%$ à l'industrie du textile. (SDI, 1984, p. 24). Ce qui manifestement reflète mal les énoncées du programme.

La Société Générale de Financement constitue avec la SDI, un important outil financier de développement industriel. Créée en 1962, elle a été pendant les dix premières années de fonctionnement, une société mixte dont l'action se réduisait à sauver les entreprises autochtones en péril. À partir de 1972, devenue exclusivement une société d'État, elle change radicalement d'orientation; son objectif est alors de créer des branches industrielles 
fortes en s'associant soit avec des capitaux étranger (joint venture) apportant leur technologie, ou avec des PME québécoise innovatrices.

Parmi ces initiatives certaines favorisent plus directement la promotion de la recherche. En 1981 par exemple, on créait la société d'investissements Novacap Inc. avec trois autres partenaires, chacun investissant un million de dollars par année destiné à constituer une source de capitaux-risque au service de l'innovation dans la PME. Et dans le sillage du «virage technologique» souhaitant le développement de secteurs technologiques de pointe, notamment les biotechnologies, la SGF a formé la Société Bio Méga Inc. D'autres initiatives semblables ont été prises dans le secteur des énergies nouvelles (Nouveler Inc.), et des produits du bois, (BioSyn Inc.). Il est inutile d'insister sur l'importance de mettre à la disposition de nouvelles entreprises innovatrices des sources de capitaux-risque qui autrement rendraient leurs projets irréalisables. Le Conseil des Sciences du Canada dans son dernier rapport sur le développement industriel, en fait d'ailleurs l'une de ses plus importantes propositions d'action (Conseil des Sciences du Canada, 1984 , p. 25).

S'il est difficile d'évaluer l'impact des politiques de promotion directe de la recherche dans l'industrie pratiquées ici et ailleurs par les gouvernements, certaines études ont démontré qu'elles sont les modes de stimulation les moins efficaces. Dans 24 cas sur les 52 innovations analysées, l'aide directe gouvernementale a faiblement contribuée à la réalisation du produit. Dans 34 cas sur 52, l'aide a peu ou faiblement contribué et dans trois cas seulement l'aide a fortement contribué ou a été déterminante pour la réalisation de l'innovation ( $R$. Rothwell et $W$. Zegveld, op. cit, tableau 4.1, p. 52-53). Il convient toutefois d'être prudent sur les conclusions qu'entrainent ces études dans la mesure où ont été analysés des cas d'innovations majeures seulement. Il n'est pas démontré en effet, qu'un appui financier accordé à une entreprise 
petite ou moyenne, n'est pas nécessaire à la réalisation d'innovations mineures (adaptation de produits ou de procédés de production) telles qu'on le pratique généralement dans l'industrie canadienne et québécoise (G. Boismenu et G. Ducatenzeiler, Ottawa, 1985, 2ième partie). Quoiqu'il en soit ces mesures vont dans le sens des intentions gouvernementales de promouvoir l'innovation en «augmentant les ressources en capital par le moyen de programme d'aide directe» (Bâtir le Québec, 1980, p. 175).

Mais il est plus important de considérer la SDI et la SGF sous l'angle d'instruments d'une politique industrielle qui travaillent à la correction de la structure industrielle québécoise et au développement d'une industrie à plus forte intensité technologique. Dans ce sens ils contribuent au renforcement du potentiel technologique québécois.

\section{L'infra-structure de recherche industrielle.}

Les entreprises autochtones sont trop faibles financièrement pour mener des travaux de R-D, d'où la création en 1969 du Centre de recherche industrielle du Québec (le CRIQ). Cette initiative constitue en quelque sorte le point de départ de la prise en charge de la question technologique par le gouvernement du Québec. L'action cependant est menée dans le contexte de la forte emprise de l'Université sur le système de recherche de telle sorte que jusqu'en 1975, le CRIQ fonctionnera suivant le modèle du laboratoire universitaire, relativement peu relié à l'industrie: en 1973-1974 par exemple, 55\% de ses projets sont initiés de l'intérieur (CRIQ, 1974).

En 1975, le Conseil d'administration est transformé et les représentants universitaires écartés. On mettra désormais l'accent sur la commercialisation plutôt que sur la performance technologique. Le CRIQ devient un support de l'entreprise: en 1979$1980,80 \%$ de ses projets sont des commandites provenant de 
l'industrie. Mais le CRIQ a pris lui aussi son virage et veut faire figure de locomotive technologique. Dans son dernier plan quinquennal 1982-1987 on prévoit le développement de nouveaux axes pour lesquels on pense verser une somme globale de $85 \mathrm{M} \$$. Il s'agirait de développer le secteur CAO-FAO (conception assistée par ordinateur - fabrication assistée par ordinateur), la robotique, la micro-électronique, le traitement et la transformation de la bio-masse et les biotechnologies; un très gros programme pour un centre dont la mission est d'abord de répondre aux besoins de l'industire. Il va sans dire que son succès dépend de l'envergure de ses moyens.

Au CRIQ dont la mission est universelle, s'ajoute d'autres centres de recherche appliquée spécialisés. Nous les mentionnons: le Centre de recherche minérale (CRM) qui a pour mission de fournir à la petite industrie des services de laboratoire qu'elle ne peut financer; l'Institut de recherche en électricité (IREQ) créé en 1967 pour répondre aux besoins d'exploitation et de distribution de l'Hydro-Québec; l'Institut national de recherche scientifique (INRS), attaché à l'Université du Québec depuis sa création en 1969, constitue un organisme de recherche à finalité industrielle dans les secteurs de l'eau, l'océanologie, la santé, les télécommunications et l'urbanisation. Que ce soit par la création (par exemple le Centre de diffusion de technologies CAO-FAO et le groupe de recherche de la Société nationale de l'Amiante) ou le renforcement de centres de recherche déjà existants (celui des pâtes et papiers de l'Université du Québec à Trois-Rivières pour ne nommer que celui-là), le gouvernement à travers divers ministères ou sociétés d'État, encourage toutes les initiatives de recherches (notamment celles qui mettent en relation les ressources universitaires et industrielles) reliées aux nouvelle technologies et aux secteurs industriels prioritaires retenus par le programme d'action économique 1982-1986. 


\section{L'intégration de l'enseignement supérieur et de l'industrie}

Parmi toutes les actions récemment mises en œuvre l'adoption de la loi favorisant le développement scientifique et technique (loi 19, juin 1983) marque clairement l'intention gouvernementale d'intervenir de façon systématique dans l'affaire du développement technologique. La loi poursuit quatre objectifs dont la création du ministère de la Science et de la Technologie (ibid, chap. 11) qui a pour fonction de conseiller le gouvernement en matière de science et de technologie, de coordonner l'ensemble des actions gouvernementales et d'intervenir auprès des agents publics et privés impliqués dans cette question.

Il lui est particulièrement dévolu le soin d'harmoniser le développement de l'enseignement supérieur et de la recherche universitaire aux besoins de l'industrie; c'est dans cette perspective que la direction de l'Enseignement supérieur du ministère de l'Éducation a été dernièrement rattachée au ministère de la Science et de la Technologie pour former le nouveau ministère de l'Enseignement supérieur, de la Science et de la Technologie. Et dans le sens de cette responsabilité, celle d'assurer cette intégration, celui-ci administre de nombreux programmes. Nous les mentionnons: le programme de soutien à l'emploi scientifique dans les entreprises lequel prévoit créer, au cours des trois prochaines années, 400 postes de professionnels et techniciens dans les PME ou les entreprises du tertiaire-scientifique. En 1984-1985 ce programme disposait d'un budget de $4 \mathrm{M} \$$. Le programme de soutien au transfert de ressources scientifiques universitaires et collégiales vers l'entreprise qui vise à renforcer les liens entre le secteur de l'enseignement et l'industrie. En 19841985 , il disposait d'un budget de $2 \mathrm{M} \$$; le programme de renforcement des liaisons universités-industries et de la recherche dite de transfert cherche à accroître les projets de recherche réalisés en collaboration avec l'industrie. Son budget de 1984-1985 était de $750000 \$$. Enfin le programme de financement de stages d'étudiants dans les entreprises 
veut favoriser l'insertion des étudiants dans le milieu industriel. Il a disposé en 1984-1985 d'un budget d'un million \$(MST, Le Bulletin de la Science et de la technologie, juillet 1984). Au total, on consacrait $8 \mathrm{M} \$$ environ à l'amélioration des liens entre l'enseignement supérieur et l'industrie.

En 1983-1984 un budget de 218 M\$ était prévu pour le financement d'innombrables mesures de développement technologique (ministère de la Science et de la Technologie, op. cit. p. 26) Par rapport à 1982-1983 (74 M\$) il s'agit d'une impressionnante augmentation de $300 \%$. Dans le contexte de grandes restrictions budgétaires, il est évident que l'on fait de cette question une priorité politique et de la promotion directe de la recherche et développement, un mode d'intervention privilégié. Mais il existe d'autres moyens de protéger l'industrie locale, de stimuler son développement technique si ce n'est sa propension à l'innovation. Nous pensons au contrôle des investissements étrangers ou aux achat publics préférentiels. Ces mesures sont d'autant plus impérieuses au Québec que le marché local est étroit, les capitaux étrangers fort importants, peu bénéfiques sur le plan technologique et les barrières tarifaires sous contrôle fédéral, en voie d'abolition (P. Faucher, A. Blais, M. de la Fuente et R. Young, 1985, Conclusion)

\section{Le contrôle des investissements étrangers}

L'une des causes qui expliquent le faible potentiel technologique de l'industrie québécoise a trait à l'importance de la main mise du capital étranger. Une étude du ministère d'État aux Sciences et à la Technologie démontre clairement que l'effort de recherche des entreprises canadiennes est toujours relativement supérieur (sinon égal dans la branche des «machines») à celui des entreprises étrangères (ministère d'État aux Sciences et à la Technologie, 1979, p. 10). Suivant la thèse du Conseil des Sciences 
du Canada, cette main mise du capital étranger sur notre industrie aurait des conséquences désastreuses sur notre autonomie technologique et ultimement sur l'état de notre économie. (J. Britton et J.Gilmour op.cit). Bref une politique de contrôle des investissements étrangers par rapport à leur impact technologique apparait important dans le contexte économique canadien et québécois.

En 1974, à la suite des recommandations du rapport Gray, le gouvernement fédéral crée l'Agence canadienne d'examen des investissements étrangers et au Québec, la Direction de l'analyse et de l'évaluation des projets d'investissements étrangers, sous la responsabilité du ministère de l'Industrie et du Commerce, reçoit la responsabilité d'opérer une première sélection des projets présentés au Fédéral. Les critères sont ceux de l'Agence: un investissement pour être retenu doit produire des retombées économiques sous la forme de création d'emplois ou d'un impact technologique. En réalité $95 \%$ des projets ont réussi l'épreuve. Ils ont été refusés, ${ }^{1}$ rarement, quand de toute évidence le marché était déjà saturé. Au niveau du gouvernement fédéral entre 1979 et $1980,8 \%$ de toutes les propositions ont été rejetés et le mérite technologique, invoqué que 230 fois contre les 840 fois qu'a été invoqué celui de la création d'emplois (Green C., 1980, tableau 10.6, p. 312).

Aujourd'hui sous le nouveau gouvernement conservateur, l'Agence canadienne a changé de nom, (Investissement Canada) d'image, sinon de réalité et le Québec probablement, s'est ajusté à une nouvelle attitude d'accueil. En réalité la venue de très gros investissements étrangers est une affaire politique et le rôle de participation de la SGF, constitue une meilleure garantie de contrôle et de bénéfices pour le Québec.

\section{Les achats publics préférentiels}

Les dépenses d'achat et de construction du gouvernement et des corps publics québécois en 1980 , se chiffraient à près de 
6,5 milliards $\$$, soit 2,3 milliards $\$$ d'achats directs et de 3,9 milliards $\$$ de travaux de construction (Y. Bernier, 1980), c'est-à-dire $9 \%$ du PIB. Dans le contexte d'un marché étroit comme celui du Québec, le phénomène est important. La protection du marché public au profit de l'industrie locale semble aller de soi. En tout cas cette pratique a fait ses preuves ailleurs notamment sur le plan des retombées technologiques. En effet ces études sur l'impact des mesures gouvernementales sur la réalisation d'innovations ont montré que la demande gouvernementale a été le mode de stimulation le plus efficace: dans 38 cas sur 52, il a été une stimulant a l'innovation au moins significatif, dans 10 cas sur 52 il a été important et dans 11 cas, il a été très important (R. Rothwell et J. Zegveld, op. cit. p. 52-53).

Or à l'exception de certains corps publics (notamment HydroQuébec qui pratique une politique d'achat préférentiel depuis 1962), cette pratique est récente au Québec et un des arguments justifiant son adoption était précisément de faire contrepoids aux politiques déjà appliquées ailleurs dans d'autres provinces canadiennes, l'Ontario surtout, et d'autres pays tels les États-Unis pour qui elle constitue un puissant instrument de développement technologique depuis la dernière guerre mondiale et une puissante barrière non tarifaire pour les produits étrangers.

La politique d'achat québécoise a été adopté en janvier 1977 en vertu d'une décision du Conseil des Ministres (77-19). Les règles sont les suivantes:

- L'achat globalisé sur une base sectorielle et régionale devra être pratiqué lorsque bénéfique sur le plan économique.

- les dispositions contenues dans les cahiers de charges doivent favoriser l'utilisation de produits québécois tout en permettant l'exercice d'une saine concurrence (décision 80-139 de septembre 1980 et 80-225 de décembre 1980) 
- lorsqu'il existe dans un secteur un nombre suffisant d'entreprises québécoises pour assurer une saine concurrence, seules les entreprises québécoises seront admises à soumissionner.

- dans certains cas exceptionnels, même lorsqu'il n'existe pas un degré suffisant de compétition, une pratique similaire pourra être suivie lorsqu'exigée par l'intérêt public dans une perspective de développement industriel

- dans certains cas particuliers et pour des raisons similaires, un soumissionnaire offrant un plus haut degré de contenus québécois ou un apport économique plus important pour le Québec pourra être favorisé même si la soumission n'est pas la plus basse. (ministère de l'Industrie et du Québec, 1984, p. 22)

Ces règles doivent être suivies par l'ensemble des corps publics et para-publics et la responsabilité de leur coordination, leur planification et leur application, appartient au ministère de l'Industrie et du Commerce.

Dans une nouvelle décision du Conseil des Ministres (82352) adoptée en décembre 1982, le gouvernement réitère sa conviction de maintenir sa politique d'achat préférentiel et même d'en faire un instrument de développement technologique. Pour ce faire le Conseil a nommé sur la Commission permanente et interministérielle des achats, un représentant du secrétariat à la Science et à la Technologie (ou du ministère de la Science et de la Technologie à partir de 1983).

Les règles de la politique d'achat du gouvernement québécois sont relativement sévères, mais il est difficile d'évaluer son efficacité. Or si elle est appliquée rigoureusement, elle peut avoir un sérieux effet d'entrainement sur des branches industrielles de pointe, particulièrement les "équipements scientifique» (matériel médical et les machines de bureaux) et les «machines industrielles» (pour la fabrication de l'hydro-électricité). 


\section{La politique commerciale}

La nécessité d'ouvrir des marchés extérieurs aux produits québécois est évidente. L'étroitesse du marché local constitue un obstacle sérieux au développement économique et singulièrement au développement technologique dans la mesure ou le succès commercial d'une innovation est fortement lié au potentiel du marché. Le gouvernement québécois fait de la prospection de nouveaux marchés un élément important de son discours économique (Bâtir le Québec, op. cit. 1979 et Le Virage technologique op. cit. 1982, chap.6) et de son action.

Depuis 1975, la SDI administre un programme d'aide à l'exportation à trois volets:

- formation de consortium dont le but est d'aider différentes entreprises qui veulent développer leurs exportations en formant un consortium dans lequel la SDI devient partenaire.

- Crédit d'implantation par lequel la SDI avance des fonds sous forme de prêts sur une période de plusieurs années pour défrayer jusqu'à $80 \%$ des dépenses encourues par les exportations qui cherchent à pénétrer les marchés étrangers.

- financement des exportations qui requièrent un financement d'appoint aux fins d'exploitation de biens ou de services.

A ces programmes de soutien s'ajoute l'action du nouveau ministère du Commerce extérieur (chap. M-29.1, 12 janvier 1983) dont la fonction est de proposer au gouvernement des politiques en matière de relations économiques et commerciales. Il est notamment de sa mission de mettre en ouvre des programmes favorisant les exportations et la réalisation d'accords industriels, puis de prospecter de nouveaux marchés pour les produits québécois 
par le biais de ses délégations commerciales établies un peu partout dans le monde.

\section{Conclusion}

L'intérêt du gouvernement québécois pour la question technologique est déjà vieux d'une quinzaine d'années et plus encore si l'on songe que Hydro-Québec pratique une politique d'achat préférentiel depuis sa création en 1962. Mais l'élaboration d'un discours et la mise en œuvre d'un ensemble d'actions plus systématique, sont encore très récents: la publication en 1982 du document Le Virage technologique en marque le point tournant symbolique, et la création l'année suivante du ministère de la Science et de la Technologie, le geste décisif quant à l'intention de mettre en place une politique cohérente. Il est déjà clair cependant que l'attitude est résolument volontaire: on parle d'engager «le virage technologique». Il est clair aussi que les actions sont déterminées; pensons à la récente création de l'Agence québécoise de valorisation industrielle (AQVIR) dont le rôle comme agent de liaison, est d'activer la réalisation d'innovations jusqu'à l'étape de commercialisation; pensons surtout qu'en une année le budget affecté au développement technologique a triplé, passant de $74 \mathrm{M} \$$ à $218 \mathrm{M} \$$.

À l'instar du gouvernement fédéral, l'accent est mis sur la promotion directe de la R-D. On crée ou on renforce des centres de recherche à finalité industrielle, on cherche à intégrer l'enseignement supérieur et l'industrie, et surtout, on augmente l'aide directe à la R-D dans l'industrie. Conjuguée à l'action fédérale, le Québec, avec d'autres provinces canadiennes, est certainement le lieu au monde où le financement public de la recherche dans l'industrie est le plus généreux (L. Séguin-Dulude et R. Lacroix, op. cit. tableau 1.4). Or on sait que ce mode de stimulation déjà éprouvé ailleurs, n'est pas des plus efficaces. Il est probable toutefois 
que dans le contexte québécois où la présence de la PME est importante, cette forme d'aide soit nécessaire. Elle a certainement la vertu de diversifier les secteurs d'innovation, alors que la pratique d'une politique d'achat comme moyen privilégié d'assurer le développement technologique, provoque à coup sûr des effets plus spectaculaires, mais comporte l'inconvénient de favoriser la concentration du développement industriel et par là les disparités régionales. Il reste quand même un instrument puissant que le gouvernement québécois à intérêt à utiliser rigoureusement.

\section{Bibliographie}

BERNIER Y., «L'économie québécoise face à la concurrence extérieurs: les fondements scientifiques de la politique d'achat ", Études internationales, vol. XV, no 1, mars 1984 , p. 61- 93.

BOISMENU G. et DUCATENZEILER G., «Le Canada dans la circulation internationale de la technologie", dans Cameron D. et Houle F., Le Canada et la nouvelle division internationale du travail, Ottawa Editions de l'Université d'Ottawa, 1985.

BOISMENU G. et DUCATENZEILER G., Développement et technologie, Bibliographie, Montréal Cahier de l'ACFAS, 1984.

BRITTON J. et GILMOUR J., Le maillon le plus faible : l'aspect du sous-développement industriel au Canada, Ottawa Études de documentation, no 43, Conseil des Sciences du Canada, Ottawa, 1980.

CENTRE DE RECHERCHE INDUSTRIELLE DU QUÉBEC, Rapports annuels, 197374, 1974 et 1979-80, Québec, 1980.

DÉBAT DE LA CHAMBRE DES COMMUNES, Discours présenté par le Ministre d'État aux sciences et à la technologie, Ottawa, 7 mars 1978.

CONSEIL DES SCIENCES DU CANADA, Le développement industriel au Canada, Quelques propositions d'action, Ottawa Rapport 37, 1984.

FAUCHER P., BLAIS A., de la FUENTE M., et YOUNG R., Politique commerciale et politique industrielle au Canada 1960-80, dans CAMERON D. et HOULE F., Le Canada et la nouvelle division internale du travail, Ottawa Edition de l'Université d'Ottawa, 1985.

FREEMAN C., "Economics of Research Development ", dans SPIEGEL I., ROSING et DEREK de Soll Price, Science, Technology and Society, A Cross Disciplinary Perspective, London Sage Publication, 1977.

LALONDE M., La politique fiscale en matière de recherche et développement, document de consultation, Ottawa, 1983.

MINISTÈRE D'ÉTAT AU DÉVELOPPEMENT CULTUREL, La politique québécoise du développement culturel, Québec, 1978. 
MINISTÈRE D'ÉTAT AU DÉVELOPPEMENT CULTUREL, Un projet collectif: Énoncé d'orientation et plan d'actions pour la mise en œuvre d'une politique québécoise de la recherche scientifique, Québec, 1980.

MINISTÈRE D'ÉTAT AU DÉVELOPPEMENT ÉCONOMIQUE, Bâtir le Québec, Phase 1, Québec, 1980.

MINISTĖRE D'ÉTAT AU DÉVELOPPEMENT ÉCONOMIQUE, Le Virage technologique. Bâtir le Québec. Phase 11, Programme d'actions économiques 1982-86, Québec, 1982.

MINISTÈRE DE LA SCIENCE ET DE LA TECHNOLOGIE, Le point sur le virage technologique, Québec, 1983a.

MINISTÈRE DE LA SCIENCE ET DE LA TECHNOLOGIE, Relevé des subventions fédérales concernant la recherche et le développement industriels, 1975-1976 à 1981-1982, 4 édition, Québec, 1983b.

MINISTÈRE DE LA SCIENCE ET DE LA TECHNOLOGIE, Bulletin de la Science et de la Technologie, vol. 3, no 1. juillet 1984. Québec.

MINISTÈRE D'ÉTAT AUX SCIENCES ET À LA TECHNOLOGIE, La R-D dans l'industrie manufacturière canadienne et de propriété étrangère, Document explicatif no 9, Ottawa, 1979.

MINISTÈRE DE L'INDUSTRIE ET DU COMMERCE, La politique d'achat du Québec, Québec, 1984.

ROTHWELL R. et ZEGVELD W., Industrial Innovation and Public policy, Londres, Frances Pinter, 1981.

SÉGUIN-DULUDE et LACROIX R., Les disparités internationales dans les efforts de R-D: Une explication de la situation canadienne, Québec, FCAC, 1983.

SECRÉTARIAT AU DÉVELOPPEMENT SCIENTIFIQUE, La recherche-développement $(R$ D) industrielle au Québec, 1971-79, Québec, avril 1982.

SOCIÉTÉ DE DÉVELOPPEMENT INDUSTRIELLE, Rapports annuels 1980-81 et 198283, Québec 1982 et 1984.

STATISTIQUES CANADA, Indicateurs de l'activité scientifique, 1982-83, cat. 88-201, Ottawa. 